\title{
Evaluation of the lung immune prognostic index in advanced non- small cell lung cancer patients under nivolumab monotherapy
}

\author{
Juan Ruiz-Bañobre ${ }^{1,2}$, María C. Areses-Manrique ${ }^{3}$, Joaquín Mosquera-Martínez ${ }^{4}$, Alexandra Cortegoso ${ }^{2,5}$, \\ Francisco J. Afonso-Afonso ${ }^{1}$, Noemí de Dios-Álvarez ${ }^{6}$, Natalia Fernández-Núñez ${ }^{7}$, \\ Cristina Azpitarte-Raposeiras ${ }^{8}$, Margarita Amenedo ${ }^{9}$, Lucía Santomé ${ }^{10}$, José Luis Fírvida-Pérez ${ }^{3}$, \\ Rosario García-Campelo ${ }^{4}$, Jorge García-González ${ }^{2,5}$, Joaquín Casal-Rubio ${ }^{6}$, Sergio Vázquez ${ }^{7}$
}

${ }^{1}$ Medical Oncology Department, Complejo Hospitalario Universitario de Ferrol, Ferrol, Spain; ${ }^{2}$ Translational Medical Oncology Group (Oncomet), Health Research Institute of Santiago (IDIS), University Clinical Hospital of Santiago de Compostela, CIBERONC, Santiago de Compostela, Spain; ${ }^{3}$ Medical Oncology Department, Complejo Hospitalario Universitario de Ourense, Ourense, Spain; ${ }^{4}$ Medical Oncology Department, Complejo Hospitalario Universitario de A Coruña, A Coruña, Spain; ${ }^{5}$ Medical Oncology Department, Complejo Hospitalario Universitario de Santiago de Compostela, Santiago de Compostela, Spain; ${ }^{6}$ Medical Oncology Department, Complejo Hospitalario Universitario de Vigo, Vigo, Spain; ${ }^{7}$ Medical Oncology Department, Hospital Universitario Lucus Augusti, Lugo, Spain; ${ }^{8}$ Medical Oncology Department, Complejo Hospitalario de Pontevedra, Pontevedra, Spain; ${ }^{9}$ Medical Oncology Department, Centro Oncológico de Galicia, A Coruña, Spain; ${ }^{10}$ Medical Oncology Department, Hospital Povisa, Vigo, Spain

Correspondence to: Dr. Juan Ruiz-Bañobre, MD; Dr. Francisco J. Afonso-Afonso, MD. Medical Oncology Department, Complejo Hospitalario Universitario de Ferrol, Av. da Residencia S/N, Ferrol 15405, Spain. Email: jurruba@gmail.com; francisco.javier.afonso.afonso@sergas.es.

\begin{abstract}
The lung immune prognostic index (LIPI) has been proposed as a new categorical blood-based biomarker to select advanced non-small cell lung cancer (NSCLC) patients for anti-programmed cell death-1 (PD-1) or programmed death ligand 1 (PD-L1) therapy. In this study, we investigate for the first time to the best of our knowledge the prognostic and predictive utility of the LIPI in a multicenter nivolumab monotherapy-based cohort. We retrospectively analyzed the influence of the baseline LIPI on overall survival (OS), progression-free survival (PFS), disease control rate (DCR), and overall response rate (ORR) among 153 patients of a cohort of 188 advanced NSCLC patients treated with nivolumab in the second line of therapy or beyond. Worse LIPI was significantly associated with shorter OS in univariate [hazard ratio (HR) $=3.12$, 95\% confidence interval $(\mathrm{CI}), 2.12-4.60 ; \mathrm{P}<0.0001]$ and multivariate $(\mathrm{HR}=3.67,95 \%$ CI, 1.96-6.86; $\mathrm{P}<0.0001)$ analyses. Worse LIPI was associated with shorter PFS (HR =1.45, 95\% CI, 1.05-2.03; $\mathrm{P}=0.03)$, but this correlation did not reach statistical significance in multivariate analysis (HR =1.49, 95\% CI, 0.942.38; $\mathrm{P}=0.09)$. Worse LIPI was associated with lower DCR in univariate [odds ratio $(\mathrm{OR})=0.41,95 \% \mathrm{CI}$, 0.24-0.70; $\mathrm{P}=0.001]$ and multivariate $(\mathrm{OR}=0.44,95 \% \mathrm{CI}, 0.25-0.78 ; \mathrm{P}=0.005)$ analyses. This study confirms the utility of the LIPI in prognostication and disease control prediction in advanced NSCLC patients treated with nivolumab in the second line of therapy or beyond.
\end{abstract}

Keywords: Lung immune prognostic index (LIPI); lung cancer; immunotherapy; nivolumab; biomarkers

Submitted Jul 21, 2019. Accepted for publication Oct 12, 2019.

doi: $10.21037 /$ tlcr.2019.11.07

View this article at: http://dx.doi.org/10.21037/tlcr.2019.11.07

\section{Background}

Although lung cancer remains as the leading cause of cancer-related death worldwide (1), over the last years several advances have been made for the management of this aggressive disease. Immunotherapy, particularly the blockade of the programmed cell death-1 (PD-1)/ programmed death ligand 1 (PD-L1) axis, has been recently established as a standard treatment in the advanced nonsmall cell lung cancer (NSCLC) setting. For instance, 
nivolumab and atezolizumab have shown a median overall survival (mOS) benefit in previously treated metastatic NSCLC patients compared to docetaxel (2-4). In the same context, but focused on the PD-L1 positive NSCLC subset, pembrolizumab has also shown a mOS gain as first and second-line of therapy $(5,6)$. Moreover, either the addition of pembrolizumab or atezolizumab to standard chemotherapy in previously untreated metastatic NSCLC or of durvalumab after chemoradiotherapy in stage III NSCLC have recently demonstrated a significant improvement in survival outcomes independently of the PD-L1 status (7-10).

To date, despite the huge efforts made looking for an appropriate biomarker to guide immunotherapy candidate selection, only PD-L1 expression determined by immunohistochemistry has been validated in some clinical scenarios. Between all the anti-PD-1 or PD-L1 [PD(L)1] antibodies approved and available in the advanced NSCLC armamentarium, only pembrolizumab has focused its clinical development on the PD-L1 positivity (5). Overall, subgroup analyses of different randomized clinical trials demonstrate better outcomes in this biomarkerpositive population (2-6), but in any case, these data do not support denying this therapeutic strategy to those NSCLC patients with a PD-L1 negative status. Maybe the inherent temporospatial heterogeneity of PD-L1, together with the differences on technical issues and scoring systems, are some of the factors behind its inconsistency as a predictive biomarker (11).

In the era of precision medicine, liquid markers appear as a promising alternative to overcome the limitations of tissue-based biomarkers. Over the last years, several groups have demonstrated the prognostic and predictive value of several inflammatory-related markers, such as the neutrophil to lymphocyte ratio (NLR), derived neutrophil to lymphocyte ratio (dNLR) and more recently, the lung immune prognostic index (LIPI) (12). In 2018 Mezquita et al. (12) have described a new categorical blood-based biomarker, the LIPI, which integrating baseline dNLR and $\mathrm{LDH}$, was able to stratify NSCLC patients under anti-PD(L)1 treatment according to survival outcomes. Recognizing the importance of validating biomarkers in the real-world clinical scenario, in this study we investigate for the first time to the best of our knowledge the prognostic and predictive utility of the LIPI in a multicenter nivolumabbased cohort.

\section{Patients and methods}

\section{Study design and data collection}

We conducted a multicenter retrospective study of a cohort of 188 patients with advanced NSCLC treated with nivolumab in the second line of therapy or beyond in the context of expanded access program between August 2015 and January 2017 from 9 Galician medical centers (Figure S1).

Complete blood cell counts and LDH level at baseline before nivolumab therapy (within 30 days before the first infusion) were extracted from electronic medical records. Demographic, clinical and pathological data were also collected.

Tumour responses were assessed by the investigators according to Response Evaluation Criteria in Solid Tumors guidelines version 1.1 every $10 \pm 2$ weeks or before if for medical reasons was indicated.

This study was approved by the Galician Research Ethics Committee (GGC-NIV-2018-01) and conducted in accordance with the guidelines for Good Clinical Practice and the Declaration of Helsinki.

\section{Statistical analysis}

Disease control rate (DCR) was defined as the proportion of patients who achieved a complete or partial response and a stable disease, and overall response rate (ORR) as the proportion of patients who achieved a complete or partial response. Overall survival (OS) was calculated from the date of nivolumab initiation until death resulting from any cause or last known follow-up for patients alive. Progression-free survival (PFS) was calculated from the date of nivolumab initiation until disease progression or death resulting from any cause or last known follow-up for patients with no disease progression. Patients who died before radiologic assessment were consider as not evaluable for response. dNLR was calculated as neutrophil count/(white blood cell count - neutrophil count) and categorized as high $(\mathrm{dNLR}>3)$ or low $(\mathrm{dNLR} \leq 3)$. LIPI was calculated as previously described by Mezquita et al. (12) based on the baseline dNLR (high, 1 factor; low, 0 factors) and LDH level (> upper limit of normal, 1 factor; $\leq$ upper limit of normal, 0 factors), establishing 3 groups: good, 0 factors; intermediate, 1 factor; poor, 2 factors.

Comparisons between patient characteristics were 
performed using $\chi^{2}$ (discrete variables) and one-way analysis of variance (continuous variables). For time-to-event analyses, survival estimates were calculated by the KaplanMeier method, and groups were compared with the logrank test. The impact of the baseline LIPI on survival (PFS and OS), and DCR and ORR was assessed by Cox and logistic regression (enter method) models respectively, adjusted for baseline dNLR and LDH level, and other major covariates. All $\mathrm{P}$ values were 2-sided, and those less than 0.05 were considered statistically significant. Statistical analyses were conducted using the Medcalc version 17.9.7 (Broekstraat, Belgium).

\section{Results}

\section{Baseline characteristics and outcomes}

Baseline characteristics and outcomes of the entire cohort were described previously by Areses Manrique et al. (13). Forty-one percent $(n=77)$ of the patients had a good (0 factors) LIPI, while 33.5\% $(\mathrm{n}=63)$ and $6.9 \%(\mathrm{n}=13)$ had intermediate ( 1 factor) and poor (2 factors) LIPI respectively. Remaining patients $(\mathrm{n}=35 ; 18.6 \%)$ have not sufficient data to be classified according to the LIPI.

Between the 153 LIPI-classified patients, median OS was 12.9 months [95\% confidence interval (CI), 10.7-20.8 months] and median PFS was 5.8 months (95\% CI, 4.2-7.1 months). No significant differences were observed between the LIPI groups according to clinicopathologic characteristics (Table 1).

\section{LIPI utility in prognostication}

Median OS for poor, intermediate, and good LIPI patients was 3.4 months (95\% CI, 1.9-6.5 months), 7.3 months (95\% CI, 4.4-12.9 months), and 20.8 months (95\% CI, 14.9not reached months) respectively $(\mathrm{P}<0.0001$; Figure $1 A)$. Median PFS was 2.8 months (95\% CI, 1.8-3.9 months), 5.1 months (95\% CI, 3.2-8.5 months), 6.6 months (95\% CI, 4.7-8.7 months) for poor, intermediate and good LIPI patients respectively $(\mathrm{P}=0.07$; Figure $1 B)$.

Worse LIPI was significantly associated with shorter OS in univariate [hazard ratio (HR) $=3.12,95 \%$ CI, 2.12-4.60; $\mathrm{P}<0.0001]$ and multivariate $(\mathrm{HR}=3.67,95 \% \mathrm{CI}, 1.96-6.86$; $\mathrm{P}<0.0001$ ) analyses (Table 2).

As expected, we found that worse LIPI was also associated with shorter PFS (HR $=1.45,95 \%$ CI, 1.05-2.03; $\mathrm{P}=0.03$ ), despite the fact that this correlation did not reach statistical significance in multivariate analysis $(\mathrm{HR}=1.49$, 95\% CI, 0.94-2.38; $\mathrm{P}=0.09$ ) (Table 2).

\section{LIPI and DCR association}

DCR for poor, intermediate, and good LIPI patients was $23 \%, 46 \%$ and $66 \%$ respectively $(\mathrm{P}=0.004)$ (Figure 2 ).

ORR for poor, intermediate, and good LIPI patients was $8 \%, 29 \%$ and $30 \%$ respectively $(\mathrm{P}=0.2)$ (Figure 2 ).

Worse LIPI was associated with lower DCR in univariate [odds ratio $(\mathrm{OR})=0.41,95 \% \mathrm{CI}, 0.24-0.70 ; \mathrm{P}=0.001$ ] and multivariate $(\mathrm{OR}=0.44,95 \% \mathrm{CI}, 0.25-0.78 ; \mathrm{P}=0.005)$ analyses (Table 3). However, there was not a significant association between the LIPI and ORR (Table S1).

\section{Discussion}

With the arrival of immune checkpoint blockade (ICB) as a standard of care treatment for various cancer types, and considering that ICB benefits only to a limited proportion of patients, the development of markers for helping in candidate selection has become a priority for the oncology community.

Knowing the relevance of biomarker validation in the real-world setting, in this study we investigated the utility of the LIPI as a prognostic and predictive biomarker in a cohort of 153 patients with advanced NSCLC treated with nivolumab in the second line of therapy or beyond. We report that worse LIPI at the start of nivolumab therapy is associated with decreased OS, PFS, and DCR. To the best of our knowledge, this is the first study that evaluates the LIPI utility in a cohort of patients treated with only one specific anti-PD-1 antibody. The results of our study confirm the data previously reported by others in mixed anti-PD-(L)1 cohorts and in one pooled analysis of antiPD-L1 (atezolizumab)-based clinical trials regarding the LIPI value $(12,14,15)$. Although initially the LIPI was postulated as an ICB specific biomarker (12), this specificity was not confirmed in subsequent exploratory retrospective pooled analyses of data from advanced NSCLC clinical trials evaluating ICB $(15,16)$. In these studies, the LIPI showed its prognostic utility not only in the ICB subset but also in the chemotherapy and targeted therapy ones $(15,16)$.

Recently, the use of steroids ( $\geq 10 \mathrm{mg}$ of prednisone or equivalent) prior to anti-PD-(L) 1 monotherapy initiation has been associated with smaller ORR, PFS, and OS in NSCLC patients. Being steroids one of the major causes of neutrophilia and lymphopenia in patients with cancer, 
Table 1 Baseline clinicopathologic characteristics distribution by lung immune prognostic index (LIPI) groups

\begin{tabular}{|c|c|c|c|c|}
\hline Characteristics & Good LIPI & Intermediate LIPI & Poor LIPI & $P$ value \\
\hline Mean age (years) & 63.8 & 62.5 & 61 & 0.50 \\
\hline Sex, No. [\%] & & & & 0.31 \\
\hline Female & 15 [19] & 14 [22] & $5[38]$ & \\
\hline ECOG-PS, No. [\%] & & & & 0.49 \\
\hline 0 & 17 [22] & $8[13]$ & $1[8]$ & \\
\hline 1 & 58 [75] & 52 [83] & 12 [92] & \\
\hline 2 & $2[3]$ & $2[3]$ & $0[0]$ & \\
\hline Current & 38 [49] & $25[40]$ & $7[54]$ & \\
\hline Former & 32 [42] & $35[56]$ & $4[31]$ & \\
\hline Never & 7 [9] & $3[5]$ & $2[15]$ & \\
\hline Histology, No. [\%] & & & & 0.33 \\
\hline Squamous & 28 [36] & 21 [33] & $2[15]$ & \\
\hline Nonsquamous & 49 [64] & 42 [67] & $11[85]$ & \\
\hline TNM stage at diagnosis, No. [\%] & & & & 0.64 \\
\hline IIIB & 27 [35] & 19 [30] & $3[23]$ & \\
\hline 1 & 49 [64] & 39 [62] & 7 [54] & \\
\hline 2 & 15 [19] & 15 [24] & 5 [38] & \\
\hline 3 & 8 [10] & $4[6]$ & 1 [8] & \\
\hline 4 & $3[4]$ & 2 [3] & $0[0]$ & \\
\hline 5 & 2 [3] & 3 [5] & $0[0]$ & \\
\hline
\end{tabular}

ECOG-PS, Eastern Cooperative Oncology Group Performance Status; NA, not available.

their use, through the increase of the dNLR (17), can be reflected in a LIPI worsening. This situation, among others, illustrates the huge potential of the LIPI as an inexpensive real-time biomarker to complement other static markers such as PD-L1 or even tumor mutation burden (TMB).

As with PD-L1, the role of TMB as a predictive biomarker for ICB has been retrospectively evaluated in different clinical trials, but conclusive studies confirming its clinical significance are needed (18). With the results of our study, together with the body of evidence available to date, retrospective testing of the LIPI in all the ICB-based randomized clinical trials already run looks mandatory. If 
A

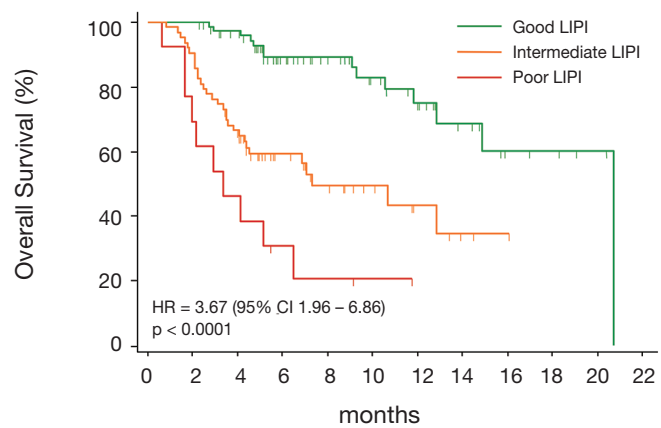

No. at Risk

$\begin{array}{lllllllllllll}\text { Good LIPI } & 77 & 77 & 68 & 41 & 33 & 24 & 17 & 10 & 5 & 4 & 2 & 0\end{array}$

Intermediate LIPI $63 \begin{array}{llllllllllll}57 & 41 & 22 & 14 & 9 & 5 & 2 & 1 & 0 & 0 & 0\end{array}$

$\begin{array}{lllllllllllll}\text { Poor LIPI } & 13 & 9 & 6 & 3 & 2 & 1 & 0 & 0 & 0 & 0 & 0 & 0\end{array}$
B

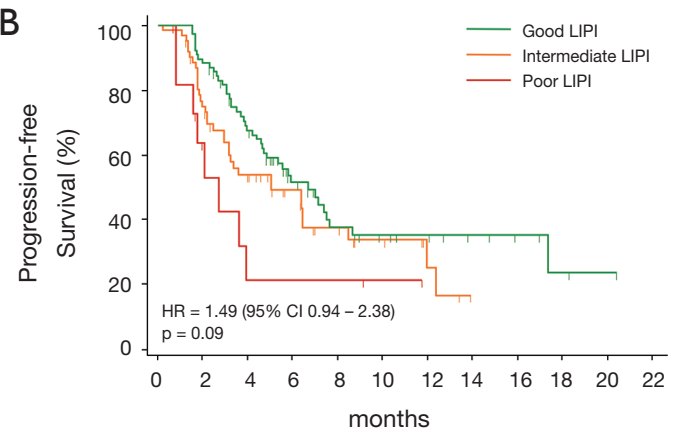

No. at Risk

$\begin{array}{lllllllllllll}\text { Good LIPI } & 77 & 68 & 48 & 25 & 16 & 12 & 9 & 6 & 4 & 2 & 1 & 0\end{array}$

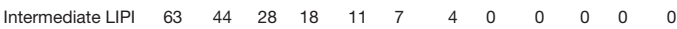

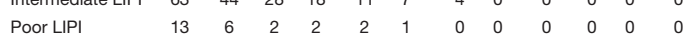

Figure 1 Kaplan-Meier overall survival and progression-free survival curves according to lung immune prognostic index (LIPI).

Table 2 Association between lung immune prognostic index (LIPI) and survival

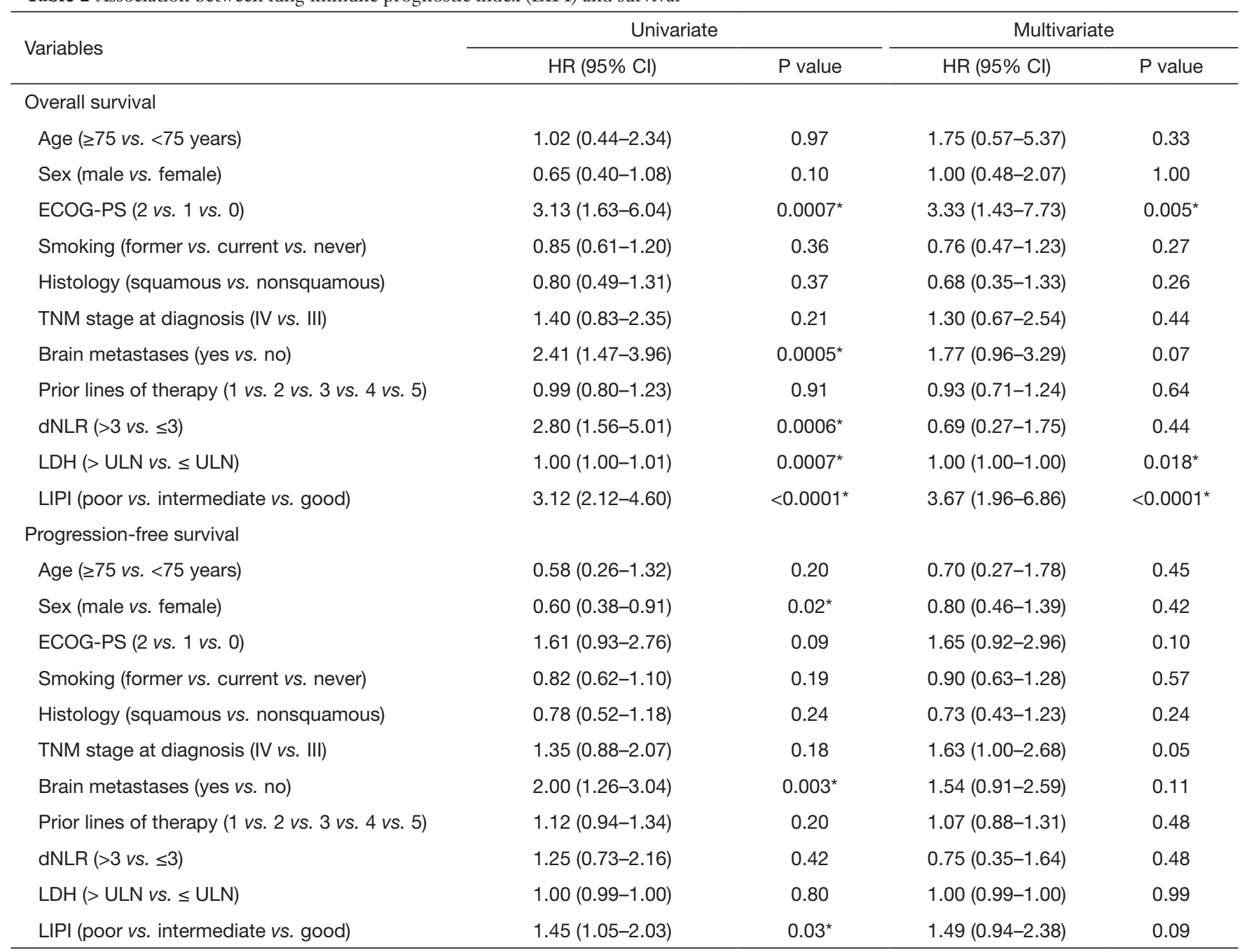

HR, hazard ratio; CI, confidence interval; ECOG-PS, Eastern Cooperative Oncology Group Performance Status; dNLR, derived neutrophil to lymphocyte ratio; LDH, lactate dehydrogenase; ULN, upper limit of normal. *, $\mathrm{P}<0.05$ indicates statistically significant. 
its role in prognostication and/or prediction is confirmed in randomized cohorts, and following the renal-cell carcinoma (RCC) experience with the International Metastatic RCC Database Consortium risk score, the LIPI should be integrated into the design of coming clinical trials as a

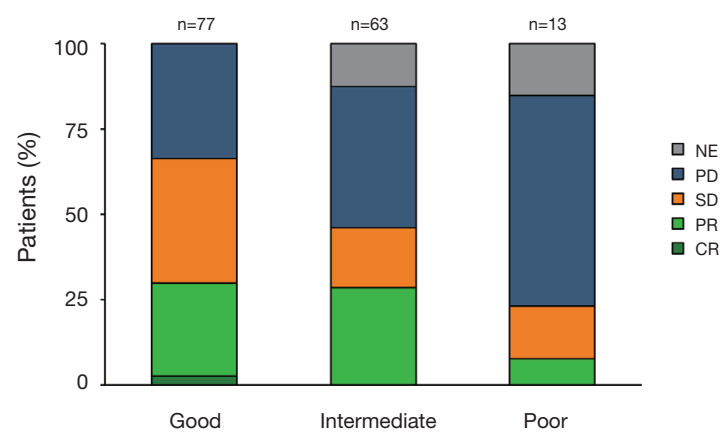

Figure 2 Nivolumab response distribution by lung immune prognostic index (LIPI) groups. NE, not evaluable; PD, progressive disease; $\mathrm{SD}$, stable disease; $\mathrm{PR}$, partial response; $\mathrm{CR}$, complete response. stratification or inclusion criterion.

We acknowledge that one of the limitations of our study, together with the limited sample size, is its retrospective nature. Due to the lack of baseline LDH level in several cases, the LIPI was only calculable for $82 \%$ of patients from the entire cohort. On the other hand, being unnecessary the PD-L1 status for prescribing nivolumab in the context of the expanded access program, it was not available for any case. Furthermore, as LDH determination was not centrally performed, methodology and reference range vary between laboratories. Finally, as the radiological assessment was performed locally with a time range of \pm 2 weeks, PFS estimation can be influenced by this variability.

In summary, the results of our study confirm the utility of the LIPI in prognostication and disease control prediction in advanced NSCLC patients treated with nivolumab in the second line of therapy or beyond. Further retrospective and prospective analyses of the LIPI in anti-PD-(L)1-based randomized clinical trials are warranted.

Table 3 Association between lung immune prognostic index (LIPI) and disease control rate

\begin{tabular}{|c|c|c|c|c|}
\hline Variable (disease control rate) & \multicolumn{2}{|c|}{ Univariate } & \multicolumn{2}{|c|}{ Multivariate } \\
\hline Age ( $\geq 75$ vs. $<75$ years) & $1.39(0.52-3.77)$ & 0.51 & $1.09(0.33-3.62)$ & 0.89 \\
\hline Sex (male vs. female) & $1.65(0.83-3.28)$ & 0.15 & $1.21(0.46-3.18)$ & 0.53 \\
\hline ECOG-PS (2 vs. 1 vs. 0) & $0.34(0.15-0.77)$ & $0.006^{*}$ & $0.33(0.12-0.88)$ & $0.03^{*}$ \\
\hline Histology (squamous vs. nonsquamous) & $0.91(0.50-1.67)$ & 0.77 & $1.24(0.56-2.75)$ & 0.59 \\
\hline TNM stage at diagnosis (IV vs. III) & $0.79(0.42-1.47)$ & 0.45 & $0.60(0.28-1.28)$ & 0.19 \\
\hline Brain metastases (yes vs. no) & $0.44(0.22-0.89)$ & $0.02^{*}$ & $0.41(0.16-1.03)$ & 0.06 \\
\hline Prior lines of therapy ( 1 vs. 2 vs. 3 vs. 4 vs. 5) & $0.90(0.67-1.20)$ & 0.47 & $0.95(0.67-1.35)$ & 0.78 \\
\hline
\end{tabular}

OR, odds ratio; $\mathrm{Cl}$, confidence interval; ECOG-PS, Eastern Cooperative Oncology Group Performance Status. *, $\mathrm{P}<0.05$ indicates statistically significant. 


\section{Acknowledgments}

The authors thank Raquel Romero Van der Schoot for technical support in data management.

\section{Footnote}

Conflicts of Interest: Juan Ruiz-Bañobre - Travel, Accommodations, Expenses: Bristol-Myers Squibb, Merck Sharp \& Dohme, Ipsen, PharmaMar. Speakers' Bureau: Roche. María C. Areses-Manrique - Consulting or Advisory Role: Roche/Genentech, AstraZeneca, Boehringer Ingelheim, Merck Sharp \& Dohme, Takeda, Lilly, Bristol-Myers Squibb. Francisco J. Afonso-Afonso - Consulting or Advisory Role: Merck Sharp \& Dohme, AstraZeneca, Bristol-Myers Squibb, Pfizer, Novartis, Takeda, Boehringer Ingelheim. Travel, Accommodations, Expenses: Bristol-Myers Squibb, Merck Sharp \& Dohme, Pfizer. Margarita Amenedo - Consulting or Advisory Role: Boehringer Ingelheim, Clovis Oncology. Speakers' Bureau: AstraZeneca; PharmaMar, Roche, Pierre Fabre, Lilly. Travel, Accommodations, Expenses: Roche, Lilly. José Luis Fírvida-Pérez - Consulting or Advisory Role: Roche/Genentech, AstraZeneca, Boehringer Ingelheim, MSD Oncology, Takeda, Lilly, Bristol-Myers Squibb. Rosario García-Campelo - Consulting or Advisory Role: Roche/Genentech, MSD Oncology, AstraZeneca, BristolMyers Squibb, Pfizer, Novartis, Takeda, Boehringer Ingelheim. Speakers' Bureau: Roche, AstraZeneca, BristolMyers Squibb, Pfizer, Novartis, Takeda, Boehringer Ingelheim, MSD Oncology. Jorge García-González Consulting or Advisory Role: Bristol-Myers Squibb, MSD Oncology, Roche/Genentech, Lilly, Boehringer Ingelheim, AstraZeneca, Pierre Fabre. Travel, Accommodations, Expenses: Bristol-Myers Squibb, Merck Sharp \& Dohme, Roche/Genentech. Sergio Vázquez - Consulting or Advisory Role: Pfizer, Astellas, Janssen, MSD Oncology, Bayer, Roche, Bristol-Myers Squibb, Boehringer Ingelheim, AstraZeneca, Ipsen, Novartis, Eusa Pharma, Eisai and Sanofi. Speakers' Bureau: Lilly, Astellas, Bayer, Roche, Boehringer Ingelheim, Ipsen, Novartis, AstraZeneca and Sanofi. Travel, Accommodations, Expenses: Pfizer, Roche and AstraZeneca. The other authors have no conflicts of interest to declare.

Ethical Statement: The authors are accountable for all aspects of the work in ensuring that questions related to the accuracy or integrity of any part of the work are appropriately investigated and resolved.

\section{References}

1. Siegel RL, Miller KD, Jemal A. Cancer statistics, 2019. CA Cancer J Clin 2019;69:7-34.

2. Borghaei H, Paz-Ares L, Horn L, et al. Nivolumab versus Docetaxel in Advanced Nonsquamous Non-Small-Cell Lung Cancer. N Engl J Med 2015;373:1627-39.

3. Brahmer J, Reckamp KL, Baas P, et al. Nivolumab versus Docetaxel in Advanced Squamous-Cell Non-Small-Cell Lung Cancer. N Engl J Med 2015;373:123-35.

4. Rittmeyer A, Barlesi F, Waterkamp D, et al. Atezolizumab versus docetaxel in patients with previously treated non-small-cell lung cancer (OAK): a phase 3, openlabel, multicentre randomised controlled trial. Lancet 2017;389:255-65.

5. Reck M, Rodríguez-Abreu D, Robinson AG, et al. Pembrolizumab versus Chemotherapy for PD-L1Positive Non-Small-Cell Lung Cancer. N Engl J Med 2016;375:1823-33.

6. Herbst RS, Baas P, Kim DW, et al. Pembrolizumab versus docetaxel for previously treated, PD-L1-positive, advanced non-small-cell lung cancer (KEYNOTE-010): a randomised controlled trial. Lancet 2016;387:1540-50.

7. Paz-Ares L, Luft A, Vicente D, et al. Pembrolizumab plus Chemotherapy for Squamous Non-Small-Cell Lung Cancer. N Engl J Med 2018;379:2040-51.

8. West H, McCleod M, Hussein M, et al. Atezolizumab in combination with carboplatin plus nab-paclitaxel chemotherapy compared with chemotherapy alone as firstline treatment for metastatic non-squamous non-smallcell lung cancer (IMpower130): a multicentre, randomised, open-label, phase 3 trial. Lancet Oncol 2019;20:924-37.

9. Gandhi L, Rodríguez-Abreu D, Gadgeel S, et al. Pembrolizumab plus Chemotherapy in Metastatic Non-Small-Cell Lung Cancer. N Engl J Med 2018;378:2078-92.

10. Antonia SJ, Villegas A, Daniel D, et al. Overall Survival with Durvalumab after Chemoradiotherapy in Stage III NSCLC. N Engl J Med 2018;379:2342-50.

11. Adam J, Le Stang N, Rouquette I, et al. Multicenter harmonization study for PD-L1 IHC testing in non-smallcell lung cancer. Ann Oncol 2018;29:953-8.

12. Mezquita L, Auclin E, Ferrara R, et al. Association of the Lung Immune Prognostic Index With Immune Checkpoint Inhibitor Outcomes in Patients With Advanced Non-Small Cell Lung Cancer. JAMA Oncol 
2018;4:351-7.

13. Areses Manrique MC, Mosquera Martínez J, García González J, et al. Real world data of nivolumab for previously treated non-small cell lung cancer patients: a Galician lung cancer group clinical experience. Transl Lung Cancer Res 2018;7:404-15.

14. Mielgo-Rubio X, Sereno Moyano M, Sotelo-Lezama M, et al. P1.01-69 Validation of the Lung Immune Prognostic Index (LIPI): Useful to Identify Resistance to PD-1 Checkpoint Inhibitors in Pretreated Lung Cancer. J Thorac Oncol 2018;13:S488-9.

15. Sorich MJ, Rowland A, Karapetis CS, et al. Evaluation of the Lung Immune Prognostic Index for Prediction of Survival and Response in Patients Treated With

Cite this article as: Ruiz-Bañobre J, Areses-Manrique MC, Mosquera-Martínez J, Cortegoso A, Afonso-Afonso FJ, de Dios-Álvarez N, Fernández-Núñez N, Azpitarte-Raposeiras C, Amenedo M, Santomé L, Fírvida-Pérez JL, García-Campelo R, García-González J, Casal-Rubio J, Vázquez S. Evaluation of the lung immune prognostic index in advanced non-small cell lung cancer patients under nivolumab monotherapy. Transl Lung Cancer Res 2019;8(6):1078-1085. doi:10.21037/tlcr.2019.11.07
Atezolizumab for NSCLC: Pooled Analysis of Clinical Trials. J Thorac Oncol 2019;14:1440-6.

16. Kazandjian D, Gong Y, Keegan P, et al. Prognostic Value of the Lung Immune Prognostic Index for Patients Treated for Metastatic Non-Small Cell Lung Cancer. JAMA Oncol 2019. [Epub ahead of print].

17. Fucà G, Galli G, Poggi M, et al. Modulation of peripheral blood immune cells by early use of steroids and its association with clinical outcomes in patients with metastatic non-small cell lung cancer treated with immune checkpoint inhibitors. ESMO Open 2019;4:e000457.

18. Chan TA, Yarchoan M, Jaffee E, et al. Development of tumor mutation burden as an immunotherapy biomarker: utility for the oncology clinic. Ann Oncol 2019;30:44-56. 


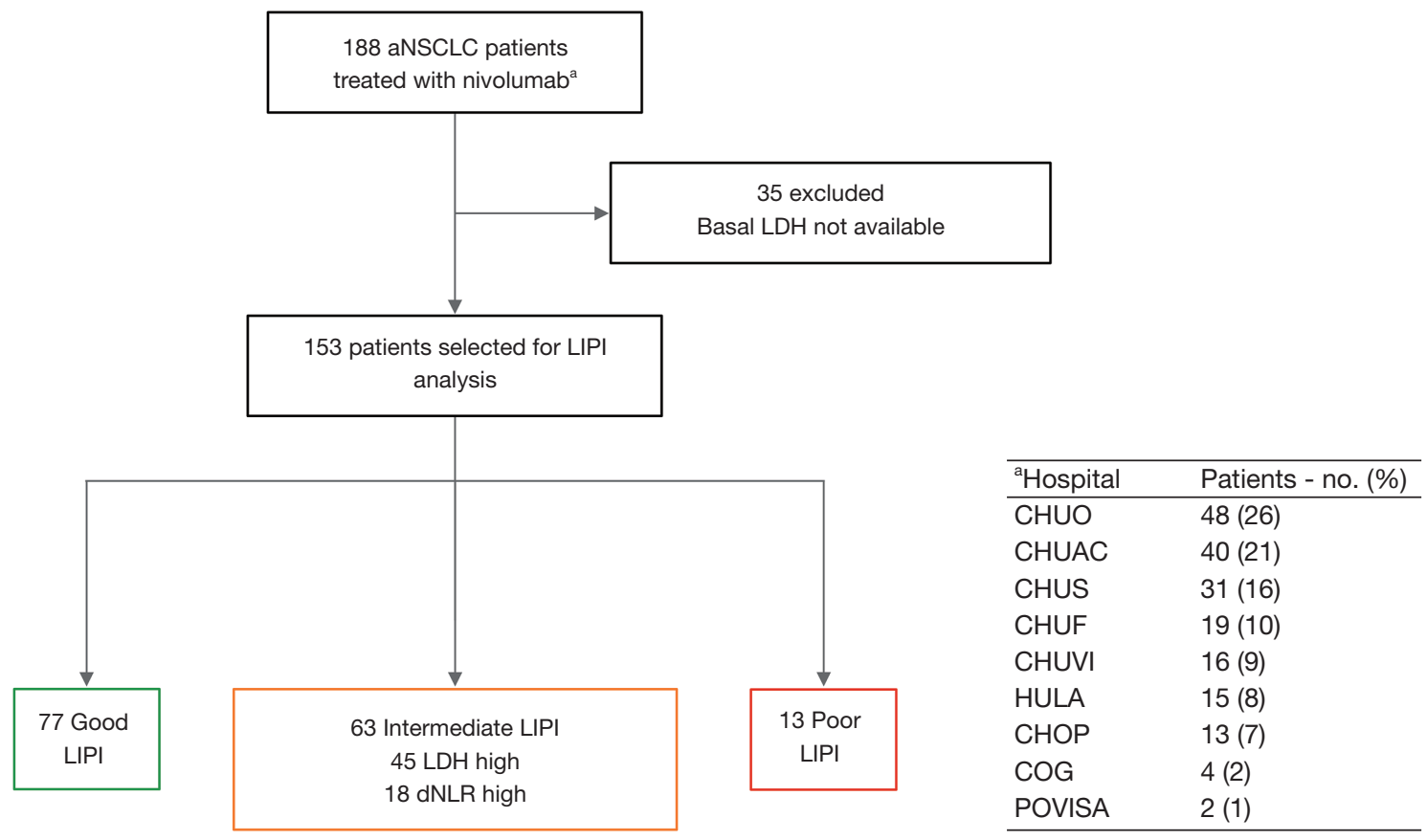

Figure S1 Flow diagram of the patient selection process. aNSCLC, advanced non-small cell lung cancer; LDH, lactate dehydrogenase; LIPI, lung immune prognostic index; dNLR, derived neutrophil to lymphocyte ratio. CHUO, Complejo Hospitalario Universitario de Ourense; CHUAC, Complejo Hospitalario Universitario de A Coruña; CHUS, Complejo Hospitalario Universitario de Santiago de Compostela; CHUF, Complejo Hospitalario Universitario de Ferrol; CHUVI, Complejo Hospitalario Universitario de Vigo; HULA, Hospital Universitario Lucus Augusti; CHOP, Complejo Hospitalario de Pontevedra; COG, Centro Oncológico de Galicia; POVISA, Hospital Povisa.

Table S1 Association between lung immune prognostic index (LIPI) and overall response rate

\begin{tabular}{|c|c|c|c|c|}
\hline Variable (overall response rate) & \multicolumn{2}{|c|}{ Univariate } & \multicolumn{2}{|c|}{ Multivariate } \\
\hline Age ( $\geq 75$ vs. $<75$ years) & $1.41(0.50-3.98)$ & 0.52 & $1.57(0.48-5.13)$ & 0.45 \\
\hline Sex (male vs. female) & $1.28(0.58-2.84)$ & 0.54 & $0.84(0.30-2.37)$ & 0.75 \\
\hline ECOG-PS (2 vs. 1 vs. 0$)$ & $0.69(0.31-1.54)$ & 0.37 & $0.77(0.31-1.93)$ & 0.58 \\
\hline Histology (squamous vs. nonsquamous) & $0.94(0.48-1.87)$ & 0.87 & $0.96(0.41-2.22)$ & 0.92 \\
\hline TNM stage at diagnosis (IV vs. III) & $0.71(0.36-1.42)$ & 0.34 & $0.54(0.25-1.17)$ & 0.12 \\
\hline Brain metastases (yes vs. no) & $0.47(0.19-1.14)$ & 0.09 & $0.56(0.19-1.62)$ & 0.29 \\
\hline Prior lines of therapy ( 1 vs. 2 vs. 3 vs. 4 vs. 5) & $0.80(0.56-1.16)$ & 0.25 & $0.84(0.56-1.27)$ & 0.41 \\
\hline
\end{tabular}

OR, odds ratio; $\mathrm{Cl}$, confidence interval; ECOG-PS, Eastern Cooperative Oncology Group Performance Status. 